\title{
Decontaminate Feature for Tracking: Adaptive Tracking via Evolutionary Feature Subset
}

\author{
Qiaoyuan Liu ${ }^{\mathrm{a}}$, Yuru Wang*a ${ }^{*}$ Minghao Yin ${ }^{\mathrm{a}}$, Jinchang Ren ${ }^{\mathrm{b}}$, Ruizhi $\mathbf{L i}^{\mathrm{a}}$ \\ ${ }^{a}$ Northeast Normal University, Changchun, P.R.China, 130117 \\ ${ }^{\mathrm{b}}$ Dept. of Electronic and Electrical Engineering, University of Strathclyde, Glasgow, U.K.
}

\begin{abstract}
Although various visual tracking algorithms have been proposed in the last 2-3 decades, it remains a challenging problem for effective tracking with fast motion, deformation, occlusion et al. Under complex tracking conditions, most tracking models are not discriminative and adaptive enough. When the combined feature vectors are inputted to the visual models, this may lead to redundancy caused low efficiency and ambiguity caused poor performance. In this paper, an effective tracking algorithm is proposed to decontaminate features for each video sequence adaptively, where the visual modeling is treated as an optimization problem from the perspective of evolution. Every feature vector is compared to a biological individual and then decontaminated via classical evolutionary algorithms. With the optimized subsets of features, "Curse of Dimensionality" has been avoided whilst the accuracy of the visual model has been improved. The proposed algorithm has been tested on several publicly available datasets with various tracking challenges and benchmarked with a number of state-of-the-art approaches. The comprehensive experiments have demonstrated the efficacy of the proposed methodology.
\end{abstract}

Keywords: Visual Tracking, Feature Subset, Decontaminate, Evolutionary algorithm.

*Yuru Wang, wangyr915@nenu.edu.cn

\section{Introduction}

Visual tracking aims to estimate the path of a target in an image sequence, given its initial location only. This technology is widely used in many applications[1][2]. The generic modes of visual tracking always extract the feature of the input image at first, then determine the target region by classification or matching methods. The features can provide a representation model of the target. Based on the model, the tracker is able to discriminate the target from its background in every frame. Since the discriminative ability of a visual model is one of the key factors to tracker's performance, an exact representation model could lead to a high accuracy for a tracker.

The targets various a lot under different visual conditions. For example, intuitively observing the magic cube shown in Fig.1(a), it can be distinguished easily due to the color difference to its background. In comparison, the texture features show more discriminative ability in Fig.1(b), and the edge features play an important role in Fig.1(c). However the tracker with specific features employed can only get good results on the specified video. That is to say, if color feature is employed, Fig.1(a) can surely be tracked well, however at the same time Fig.1(c) may meet a failure. Therefore the features used for tracking must be adaptive with the sequences' specific salient characteristic.

Investigating on the state-of-the-art trackers, the visual models employed including the single feature model and the feature integration model are all fixed with no adaptiveness to the detected feature. The single feature based models are being popular from the beginning of the 20th century until nowadays, the representative works including color [3], edge[4] and motion[5]. In addition, many generative models like sparse coding [6], PCA [7] and online density estimation [8] had also received excellent performance and became a hot issue. In recent years, the single feature based visual models are still in use and perform well. For example, Henriques [9] proposed to track via 


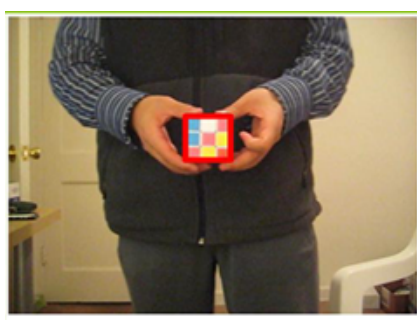

(a)

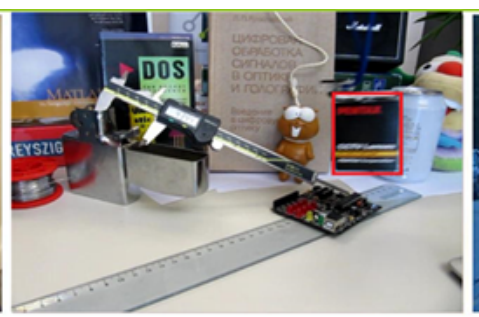

(b)

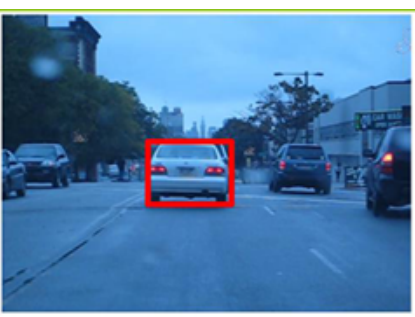

(c)

Fig 1 The salient features in different video examples.

learning and detecting over translated image patches in 2015, and standard histogram of gradients (HOG) [10] feature was employed in his Kernelized Correlation Filters (KCF) model. Zhang [11] realized a (Real-Time Compressive Tracking) CT method, in which the extracted Haar-like [12] features were compressed with a sparse measurement matrix for the appearance model. With better performance, the feature integration model is becoming more welcomed than the single feature based model in recent years. The typical work include the weighted sum model [13], hierarchical model [14], the HMM model [15], the Gaussian mixture model [16] and the pyramid model [17]. The idea of integration has also been realized in the Deep Learning framework: a STCT method [18] extracted an effective feature map via convolutional networks, the networks are trained from a large scale image classification dataset for tracking.

It has been tested that the feature integration models are superior to single feature based models, because it can comprehensively consider the characteristic in video sequences. For example, when color and texture features are employed together, the tracker can perform well on the videos in which both of these features are dominant. In contrast, the trackers only employ color features could not perform well on the sequences in which the texture feature is dominant.

However, due to diversity of video sequences, which kind of feature is absolutely dominant in tracking can not be determined easily. It is very likely to be feature A and feature B constitute the characteristics of video together. So can it be half of feature A and half of feature B? Absolutely not, perhaps 20 percent of $\mathrm{A}$ and 80 percent of $\mathrm{B}$, or 45 percent of $\mathrm{A}$ and 55 percent of $\mathrm{B}$, it depends. Different videos may need different proportions on feature construction, thus an adaptive representation model is needed. There is a saying that give what you needed is better than give you all, so we need to tailor useful information to specific video sequence. In this way lots of noise or redundant information in integration model could be decontaminated, the computation load could be alleviated and "Curse of Dimension" could be avoided. Moreover, the complexity of appearance model which constrains its further application to visual tracking could be decreased.

To the best of our knowledge, there are few researchers paying their efforts on tracking with selected information. A similar work with this idea is the method of Grabner etc. [19] in 2006, they selected the best weak classifiers corresponding to the features in their Adaboost framework. This method received a great improvement on tracking results in the early 21 th century. The success mainly relied on the adaptiveness of the boosting. Different from this method, selecting feature is treated as an optimization problem in our algorithm, the features are decontaminated directly before assigning them to the classifiers.

The goal of decontaminating features is to build an adaptive visual model for a specific video. In this paper, we resolve it as an optimization problem, while the evolutionary algorithm is em- 
ployed in selecting the optimal feature subset. Moreover, in order to keep adaptiveness of the visual model on a given feature subset over the whole sequence, we update the model parameters and feature pool in a Sequential Monte Carlo framework. Instead of generative models that focus on the target matching, we realized our research in a discriminative framework of ensemble.

The overall framework of the proposed tracker is shown in Fig.2. Specifically, a best string is obtained using Evolutionary Algorithm at the first frame (shown in the right column): decontaminate the features extracted into feature subsets using binary codes, in which the binary codes are optimized iteratively by Evolutionary Algorithm. The binary string represent the best features expression. Pass the binary string to all the follow-up frames, equal to let the tracker work with the most suitable features, train the classifiers with features in higher quality the tracking accuracy is sure to be enhanced. To illustrate the universality of our method, the most basic evolutionary algorithm Genetic Algorithm [20] is utilized as an example to obtain an optimal feature subset from the feature pool. The final solution is generated iteratively by evaluating the qualities of feature subsets using the objective function, which is defined to analysis distribution between interesting region and ground truth bounding box.

The proposed method has been tested on the widely used benchmark datasets OTB2015[21] and VOT2016[22]. Extensively experiments and discussion has been performed on representative video sequences, which cover various tracking challenges. Compared with several state-of-the-art trackers, our tracker is demonstrated to perform with better or at least comparable results.

This paper is organized as follows: We first describe the process of decontaminating features for tracking in Section 2. The detailed methodology of model updating is described in Section 3. In Section 4, the overall framework of our tracker is described. Section 5 illustrates results and discussion.

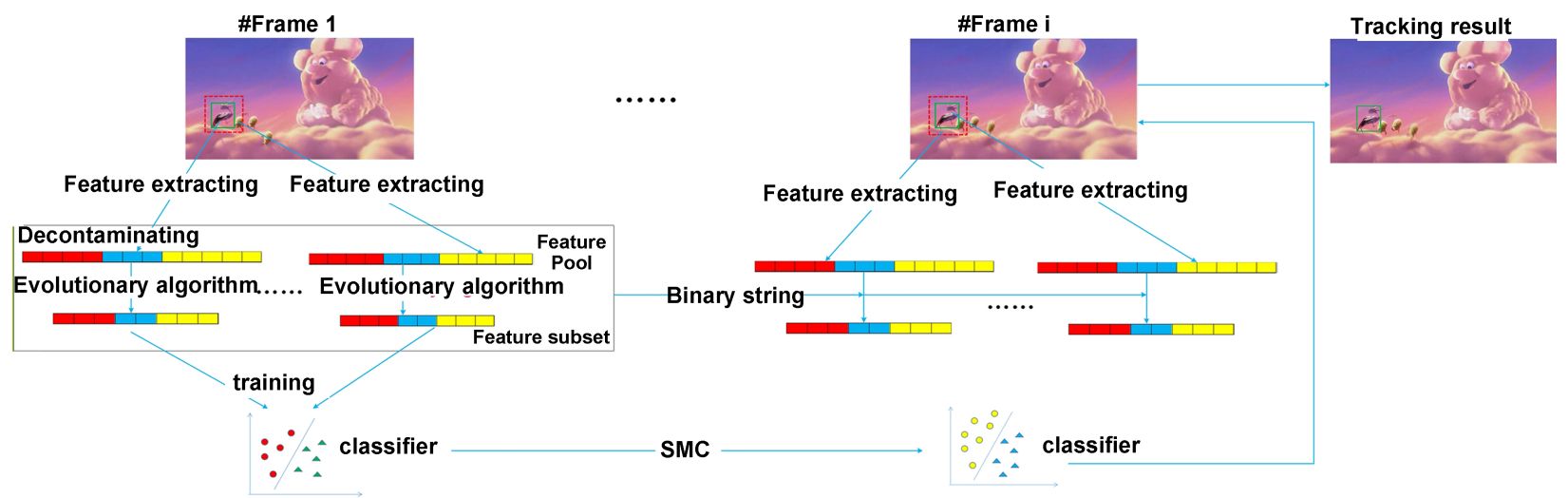

Fig 2 Overview of the proposed decontaminating feature based tracker.

\section{Decontaminate Features for Tracking}

\subsection{Feature Pool}

The bounding box in every frame is represented as a multi-scale patches model with four levels similar to pyramid representation[23]. In detail, at the first level, we divide the bounding box into patches of size $n \times n$ uniformly. At the second and third levels, larger patches that cover different 


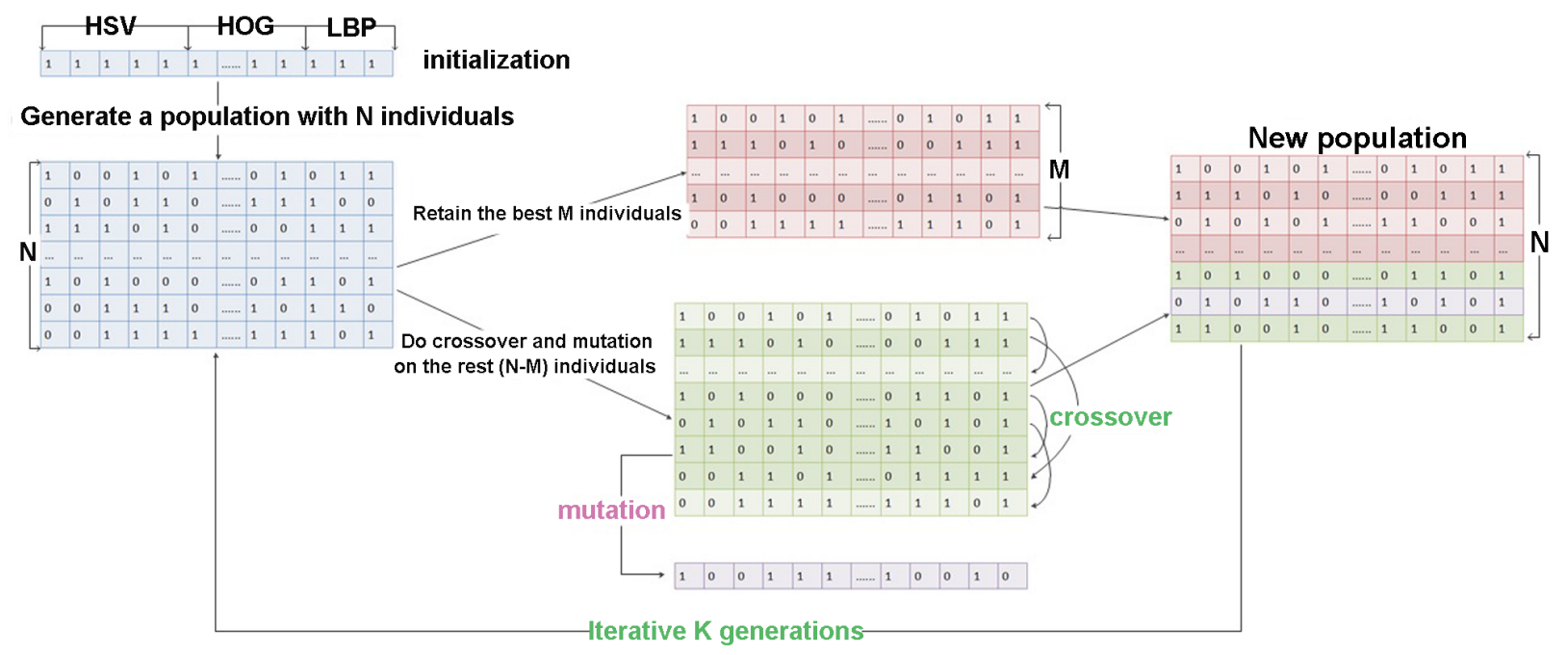

Fig 3 Approximate the optional solution by the binary evolutionary algorithm.

portions of the object are also selected by divide the bounding box into the number of $4 \times 4,2 \times$ 2 patches evenly. At the highest level, the whole bounding box is considered as one patch.

In the initial procedure, widely used features including 64-bins Hue-Saturation-Value (HSV) color/gray-scale histogram, standard histogram of gradients (HOG) and Local Binary Patterns (LBP) are extracted in the patches of four scales to form a feature combination. There exist ineffective features that are less dominant in discriminating, which are regarded as noise or redundant information. The combination with redundant information could not only effect describing the characteristic of every video sequence adaptively, but also increase the computation load and have a negative impact on tracking result. Our task is to tailor feature to every specific video adaptively and provide a subset with better discriminative ability for tracking.

\subsection{Decontaminate Feature via Evolutionary Algorithm}

In this section, we will fully introduce the method of optimizing the binary coded feature vector with evolutionary algorithm, and its solution processes.

\subsubsection{Evolutionary Method for Optimization}

Since the feature vectors with somewhat redundant information couldn't describe the characteristic of every frame adaptively, we need to remove the redundant ones in order to improve the representative ability of visual model. The evolutionary algorithm is characterized by weeding out the worst solution and leaving a better one to meet the needs of decontaminating feature for tacking precisely. Evolutionary algorithms [24][25][26] simulate the way of biological evolution, aim to gradually find an optimized solution through selection, crossover, mutation and some other ways with the objective function as optimization direction. To illustrate the universality of our method, the most basic evolutionary algorithm Genetic Algorithm is took as an example to resolve the above optimization problem, therefore we suggested that the evolutionary algorithms with more complex strategies could perform with better result. 
Every feature subset is represented as a binary string as shown in Fig.3, it is seen as an individual in the population. At each iteration step, $N$ binary strings $B=\left(b_{0}, b_{1}, \ldots, b_{m-1}\right)$ are generated randomly, $b_{i}=\{0,1\}$ (in which 0 represents remove this feature from feature vector and 1 represents retain). For each individual, the objective function will return a fitness value which estimates the discriminative ability of the feature subset. Maximize or minimize objective value $J$ in the following selection, crossover and mutation steps, a best string is to be obtained by optimizing the initial strings:

Selection: Fitness values are obtained by evaluating every individuals with objective function. Sort the corresponding fitness values, our algorithm retains the best $\mathrm{M}$ individuals as elite and leaves them to the next generation.

Crossover: For the rest (N-M) individuals, do a randomly mutual crossover according to a fixed probability. Two new individuals will be obtained in every crossover processing. Evaluate the new individuals with the objective function again, if the new individual is worse then retain the old one, otherwise replace the old with the new one.

Mutation: Choose an individual randomly from the (N-M) individuals, find the mutation point in this string according to a mutation probability and invert it. Similar to the crossover, replace the old individual with new one in better quality, otherwise discard the new string. Thus a new population is formed with the new (N-M) individuals and the elite individuals.

Iterative execute the above process for $\mathrm{K}$ generations until a best solution convergence, which represents the best feature subset for tracking.

\subsubsection{Objective Function}

As the core of optimization algorithm, objective function represents the optimization direction. With an appropriate objective function, it is sure to obtain an optimized feature subset for visual model.

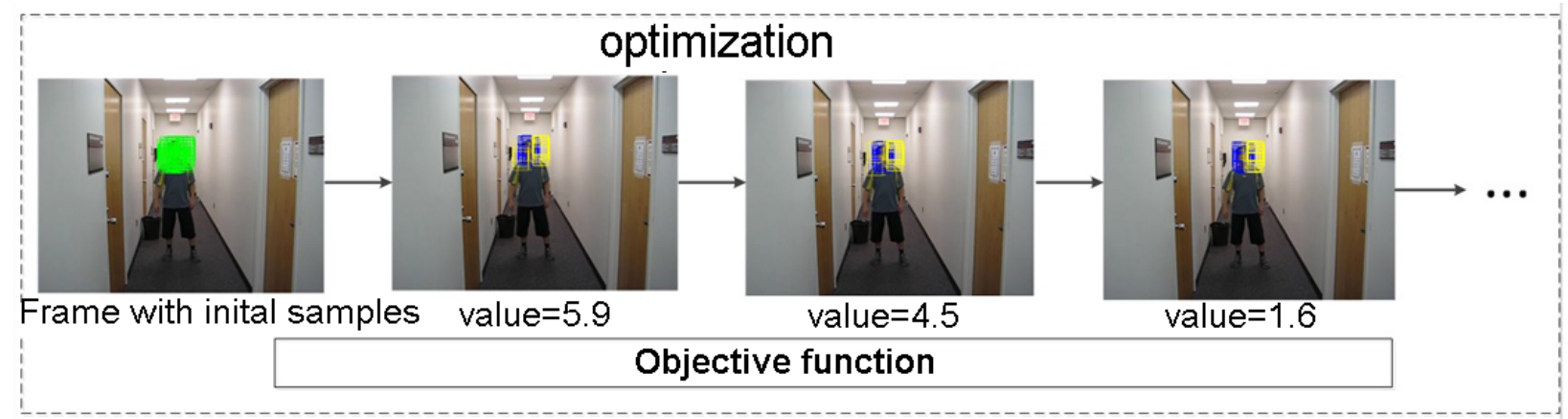

Fig 4 Schematic diagram of objective function optimization, in which the green bounding boxes in the first picture represent samples generated randomly, the blue ones in the following pictures represent the best samples, and the yellow ones represent the worst.

During the optimization procedure, in order to distinguish the target and background, a number of random samples(bounding boxes) are generated to simulate the distribution of the pixels in the target and background. Specifically, $\mathrm{N}$ samples in the detection region (including target and its neighbor background) are generated according to a Gauss perturbing on the ground-truth location, and each sample is represented as a regional image. Our task is to determine which of these samples 
belong to the target region according to the objective function. In order to prove the feasibility of the optimization algorithm in decontaminating feature, two objective functions are designed to evaluate the feature subsets from different perspectives. Fig.4 shows the random samples and the process of optimization with the objective function, specifically the second objective function is took as an example.

Object Function $J_{1}$ : Each feasible solution (random binary string) corresponds to a random combination of the feature vectors. Based on the initial features extracted from image samples and ground truth, simplified feature vectors are obtained according to the random combination strategy. Then calculate all the average distances $d_{i}, i \in 1 \ldots N$ between the simplified feature vector of samples and the simplified feature vector of ground truth bounding box according to the following formulation, specifically the ground truth of the first frame is given in benchmark for comparison:

$$
d_{i}=n^{-1} \sum_{f=1}^{n} \sqrt{d s_{f}^{2}-d g_{f}^{2}}, i=1 \ldots N
$$

where $n$ is the size of feature vector. $d s_{f}$ is the $f$-th element of this sample feature, $d g_{f}$ is the $f$-th element of the ground truth feature. Sort the distances in descending order and select the samples farthest and nearest to ground-truth to obtain worst and best mean value $d_{w}, d_{b}$ in following equation, in which $N_{w}$ and $N_{b}$ are the numbers of the worst and best samples respectively.

$$
d_{w}=N_{w}{ }^{-1} \sum_{N-N_{w}}^{N} d_{i}, d_{b}=N_{b}{ }^{-1} \sum_{1}^{N_{b}} d_{i} N_{b}
$$

Finally, the objective value is defined as:

$$
J_{1}=d_{w}-d_{b}
$$

We assume that a larger objective value represents a stronger discriminative ability of the feature subset.

Object Function $J_{2}$ : This function is designed according to the ideal of the linear discriminant analysis(LDA)[27]: it is also based on random samples. The similarity is calculated by dot product the simplified features extracted from every random sample and ground-truth. Select the best and worst random samples to build two clusters $A=\left\{A_{i} \mid i=1 \ldots N_{b}\right\}, B=\left\{B_{i} \mid i=1 \ldots N_{w}\right\}$ according to similarity, in which $N_{b}$ and $N_{w}$ are the numbers of best and worst samples, $A_{i}$ and $B_{i}$ are samples' locations in corresponding cluster respectively. Then calculate the objective value $J_{2}$ in following equation:

$$
J_{2}=\frac{S_{w}}{S_{b}}
$$

where $S_{w}$ is within-class scatter, and $S_{b}$ is between-class scatter. $S_{w}$ is calculated by the sum the covariances of selected samples in two clusters.

$$
S_{w}=\operatorname{cov}(A)+\operatorname{cov}(B)
$$

$S_{b}$ is the Euclidean distance between the best mean center location $\bar{A}$ and worst mean center location $\bar{B}$. The smaller the obtained fitness value is, the more effective for tracking the feature 
subset is.

$$
S_{b}=\sqrt{\bar{A}^{2}-\bar{B}^{2}}
$$

In the above evolutionary algorithm, all the features are binary coded and optimized in the way of biological evolution. After the evolution, apply the optimal solution to the feature combination, it will be decontaminated with the character of optimal discriminative ability and efficiency.

\section{Model Updating}

A tracker often has to adapt to significant changes in tracking scenarios. Therefore a fixed visual model on a given feature subset may not sufficient to keep adaptiveness over the whole sequence. In order to improve the adaptability, we proposed to update the tracking parameters in a Sequential Monte Carlo (SMC) framework.

Know that the discriminative model possesses better robustness, we realized the above visual model in an ensemble tracking framework with hybrid classifiers. Every detection region is divided into a pyramid with patches in four scales as mentioned in section 2.1. Each image patch is corresponding to a weak classifier, the smaller patches in regular size $n \times n$ are assigned to LDM classifiers, the larger patches in other sizes are assigned to SVM classifiers. Scale-adaptive hybrid weak classifiers are weighted combined as a strong one. And the final results are output from the strong classifier. You can find details in our previous work[28].

In the tracking process, the features of detection region would undergo great changes. To improve adaptiveness and generality of the tracker, both the weight vectors and the pool of weak classifiers are updated by frame to evolve the model.

The sequentially arriving flow of weight vector $V_{t}$ over the whole video sequence is seen as a state-evolving procedure. From a Bayesian point of view, its updating is estimated by obtaining its posterior distribution.

Given a sequence and a corresponding sequence of observations, the goal is to find a max posterior distribution. The Monte Carlo method provides a possible solution to this problem . Particle Filter (PF)[29] is a recursive Bayesian filter that belongs to the SMC methods.

According to the classical PF, at time $t-1$, the posterior probability distribution $p\left(v_{t-1} \mid Z_{t-1}\right)$ is usually approximated by using a finite number $(N)$ of weighted $w_{t-1}^{i}$ samples $p\left(v_{t-1} \mid Z_{t-1}\right) \approx$ $\left\{w_{t-1}^{i}, v_{t-1}^{i}\right\}_{i=1}^{N}$. In our methods, $\mathrm{N}$ is set to 500 specially. Then, the posterior distribution $p\left(v_{t} \mid Z_{t}\right)$ can be approximated by using some weighted samples as

$$
p\left(v_{t} \mid Z_{t}\right) \approx c p\left(Z_{t} \mid v_{t}\right) \sum_{i=1}^{N} w_{t-1}^{i} p\left(v_{t-1}^{i} \mid Z_{t-1}^{i}\right)
$$

Because it is difficult to draw samples from the posterior distribution, the important sampling method is usually performed by a proposal distribution. Samples are drawn from a proposal density $q$ as

$$
v_{t}^{i} \sim q\left(v_{t}^{i}\right) \triangleq \sum_{i} w_{t-1}^{i} p\left(v_{t}^{i} \mid v_{t-1}^{i}\right)
$$

The sample weight is usually recursively updated as

$$
w_{t}^{i}=p\left(Z_{t-1}^{i} \mid v_{t-1}^{i}\right) w_{t-1}^{i}
$$


The weight of each weak classifier is updated based not only on the observation at the current frame but also on its consistency with the adjacent frames. In such a way, abrupt changes will be avoided and reliable updating is realized.

\section{Overview of the Adaptive Tracker}

Above all, in order to decontaminate the redundant information from the feature pool, we propose an adaptive visual model. An optimal feature subset is selected using evolutionary algorithm, the adaptiveness is kept by modeling the parameters in a SMC framework. This adaptive visual model is realized in an ensemble tracking framework. The overall algorithm is shown in Algorithm 1.

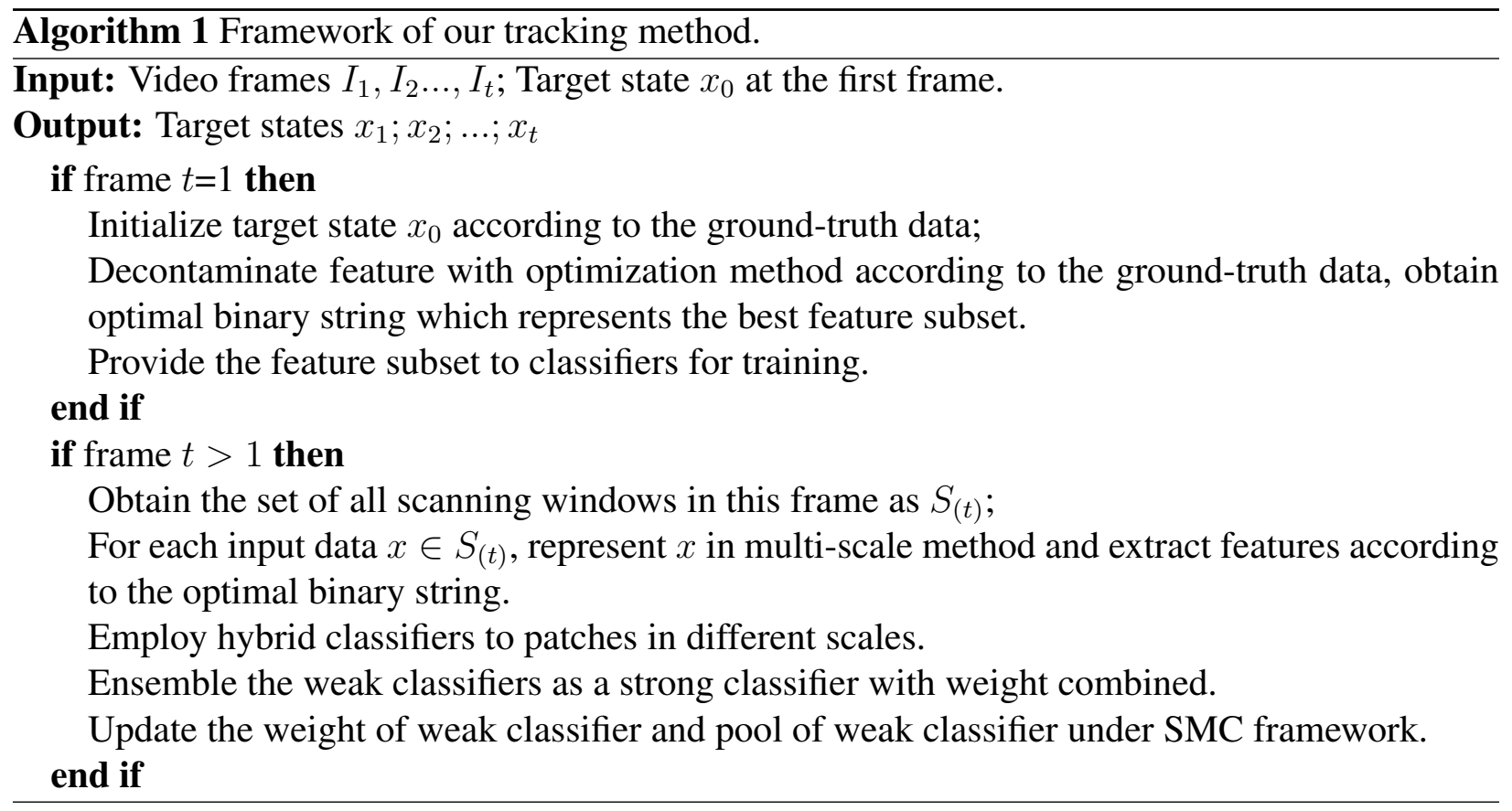

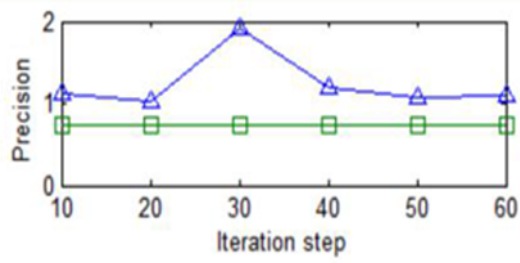

(a)

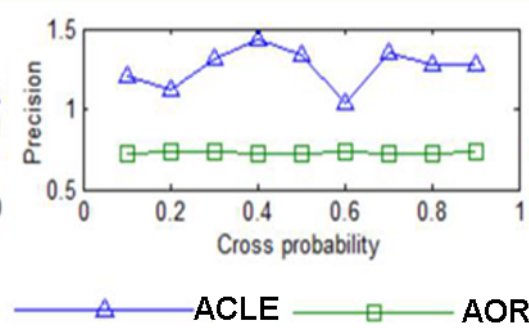

(b)

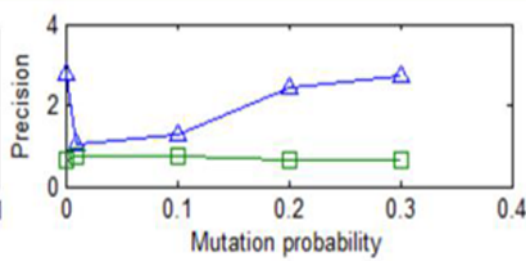

(c)

Fig 5 The curves of the parameter settings on tracking performance.

\section{Results and Discussion}

In this section the implementation details and parameter settings are described. 
There are few benchmarks for visual tracking proposed[21,22]. Considering the fairness, the effectiveness of our tracking method is demonstrated by quantitative and qualitative analysis not only on the widely used dataset OTB2015[21] with various challenges, including illumination variation, scale variation, occlusion, deformation, in-plane rotation, out-of-plane rotation, background clutters, and low resolution but also on rich VOT2016 datasets[22] which cover many representative datasets, such as ALOV+++[30], non-tracking datasets, Computer Vision Online, Professor Bob Fishers Image Database and some other representative datasets. Similar to popular trackers proposed in [21] visual cues including HSV, HOG and LBP are employed, the feature pool construction is already sufficient to analyse the effect of feature decontamination.

\subsection{Evaluation methodology}

We employ five widely used metrics for evaluation: Average Center Location Error (ACLE), Average Overlap Ratio (AOR), success rate, precision plots and average expected overlap.

The metric of ACLE [31] is the average Euclidean distance between center of predicted and ground truth bounding box. AOR according to the Pascal VOC criteria [32] is the overlap ratio between result output and ground truth bounding box. ACLE and AOR can evaluate the tracking method from the aspect of each video sequence frame to frame which is more intuitive. Specially, larger AOR represents better performance, and smaller ACLE represents better performance.

The success rate and precision plot follow the evaluation protocols in [33]. The ratio of successfully tracked frames is measured by a set of thresholds, where bounding box overlap ratio and center location error are employed in success rate and precision plot, respectively. We rank the tracking methods based on Area Under Curve(AUC) for success rate plot and center location error at 20 pixels for precision plot. The success plot and precision plot that consider all results of different visual sequences can provide a more comprehensive comparison.

The average expected overlap is the average overlap ratio between bounding box and the ground truth similar with AOR. For a full treatment of this metric, readers are encouraged to read [22].

\subsection{Parameters Discussion}

We study the parameter settings on the tracking performance. Overall, the controlling parameters include the number of iterations, the encoding ratio, the crossover and mutation probabilities in the GA. Since the video sequences "boy" covers most tracking challenges such as "scale variation", "motion blur", "fast motion", 'in plane rotation", "out of plane rotation", all the parameters are tested on this certain video for analysis. For each parameter, we test its influence in its definition domain when the others are fixed. The curves in Fig. 5 presents the influence of the parameters settings on the tracking performance.

The genetic algorithm starts from an initial solution, and converges to an optimal one after a number of iterations. When other parameters (crossover and mutation probabilities) are fixed, number of iterations determines the precision of the optimal solution. We test number of iterations from 10 to 60. And from the curves shown in Fig.5(a), we can see that the tracking performance, especially the AOR curves is not sensitive to the number of iterations. Overall, it is fixed to 40 in all experiments.

The mutation and crossover probabilities influence the convergence speed of GA. For the crossover probability, it is set in the range of 0.1 to 0.9 . And for the mutation probability, it is usually below 0.3 , therefore, it is set as $0.001,0.01,0.1,0.2$ and 0.3 . From the curves in Fig. 5, the 

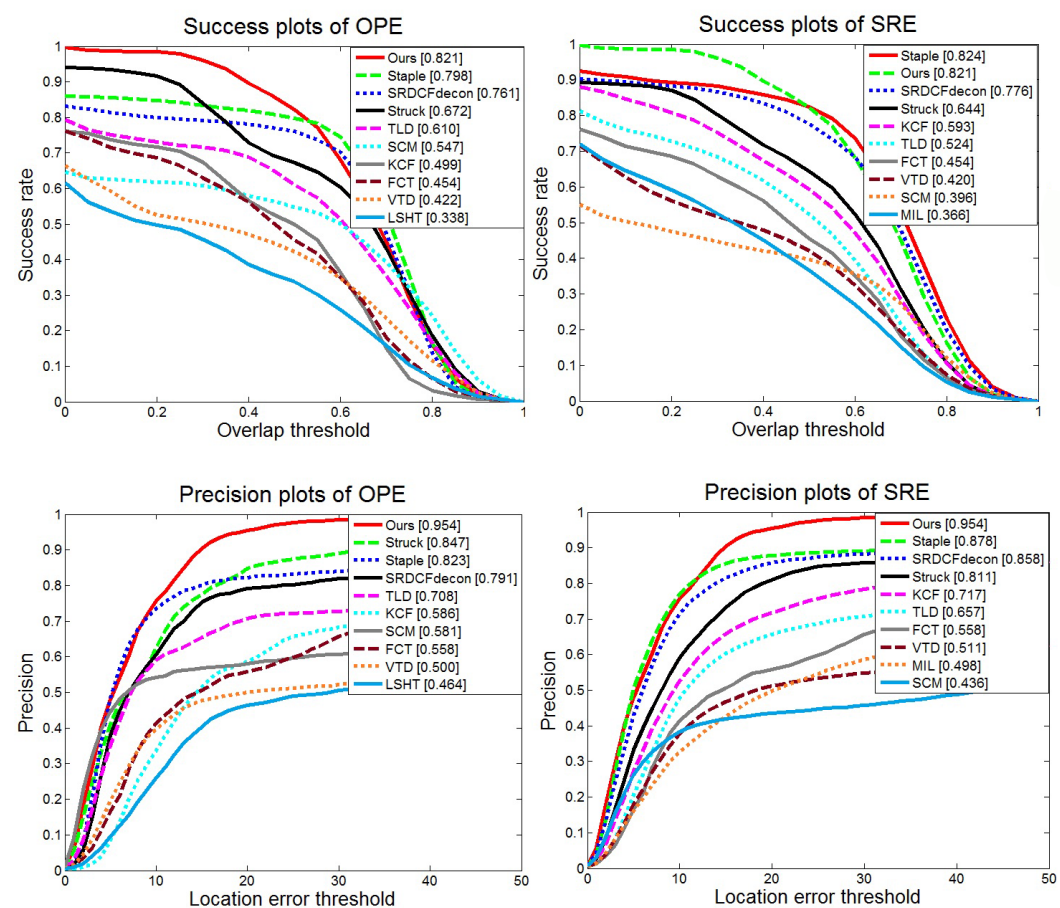

Fig 6 Average success plot and precision plot on OTB2015.

AOR metric shows less sensitiveness to these two parameters. However a big difference is shown between the ACLE curves. Specifically, the tracker reaches the minimum error when the crossover probability is around 0.6 and the mutation probability is 0.01 . That means frequent crossover or mutation will lead to premature convergence. Therefore, these two parameters are set to 0.55 and 0.01 .

Table 1 Tracking sequences used in our experiments

\begin{tabular}{|c|c|c|}
\hline Video sequences & Nubmer of frames & Main challenges \\
\hline Boy & 602 & $\begin{array}{l}\text { Scale Variation,Motion Blur,Fast Motion,In-Plane Rotation, } \\
\text { Out-of-Plane Rotation }\end{array}$ \\
\hline Basketball & 725 & $\begin{array}{l}\text { Illumination Variation, Occlusion,Deformation, Out-of-Plane } \\
\text { Rotation,Background Clutters }\end{array}$ \\
\hline Car4 & 659 & Illumination Variation,Scale Variation \\
\hline Coke & 291 & $\begin{array}{l}\text { Illumination Variation, Occlusion,Fast Motion,In-Plane } \\
\text { Rotation,Out-of-Plane Rotation,Background Clutters }\end{array}$ \\
\hline Couple & 140 & $\begin{array}{l}\text { Scale Variation,Deformation,Fast Motion, Out-of-Plane } \\
\text { Rotation,Background Clutters }\end{array}$ \\
\hline Deer & 71 & $\begin{array}{l}\text { Motion Blur,Fast Motion,In-Plane Rotation,Background } \\
\text { Clutters,Low Resolution }\end{array}$ \\
\hline Football1 & 81 & $\begin{array}{l}\text { In-Plane Rotation,Out-of-Plane Rotation,Background } \\
\text { Clutters }\end{array}$ \\
\hline Girl & 500 & $\begin{array}{l}\text { Scale Variation, Occlusion,In-Plane Rotation, Out-of-Plane } \\
\text { Rotation }\end{array}$ \\
\hline Walking2 & 500 & Scale Variation,Occlusion,Low Resolution \\
\hline Bird2 & 99 & $\begin{array}{l}\text { Occlusion,Deformation,Fast Motion,In-Plane Rotation, } \\
\text { Out-of-Plane Rotation }\end{array}$ \\
\hline
\end{tabular}




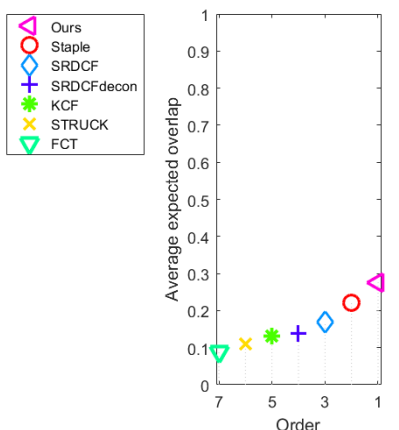

(a)

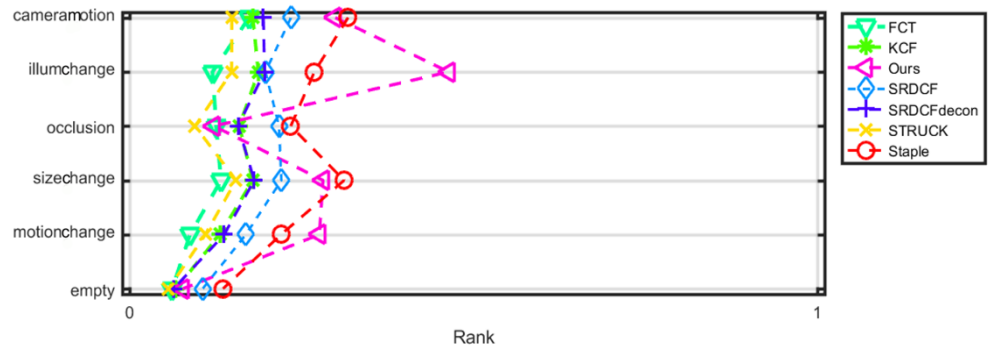

(b)

Fig 7 Average expected overlap plot on VOT2016(a). Performance rank between our method and other state-of-the-art methods under different visual challenges(b).

\subsection{Comparison with State-of-the-Art Methods}

Our method is compared with ten state-of-the-art publicly available visual trackers, including MIL [34], VTD[35], Struck [36], SCM [37], TLD[31], LSHT [38], FCT [39], KCF [9], Staple [40] and SRDCFdecon [41] on dataset OTB2015. The overall performance on OPE and SRE evaluation is shown in Fig. 6. According to the plots, we can see that the proposed approach achieves the best precision in both OPE and SRE. For success rate, ours is the best for OPE and the second best in SRE.

Besides, we also compared our method with six representative tracking methods on datasets VOT2016, including Staple [40], SRDCF[42], SRDCFdecon [41], KCF [9], FCT [39] and Struck [36](The reason for the lack of several methods is that these methods didn't provide results for VOT datasets). The overall performance on average expected overlap evaluation and the performance rank between our method and other state-of-the-art approaches under different visual challenges are shown in Fig. 7. As seen, our method reaches a higher average expected overlap ratio and on-average ranks the first under various visual challenges, especially in coping with illumination changes.

Since in both datasets, our method performs with better or at least comparable results, to evaluate intuitively, we select ten video sequences from the OTB2015 benchmark dataset. The selected sequences cover various attributes in tracking challenges are shown in Table 1, the AOR and ACLE results compared with six representative tracking methods are reported in Table 2 and Table. 3 . The proposed tracker with two different objective functions Ours $\left(J_{1}\right)$ and Ours $\left(J_{2}\right)$ (described in formula(3) and (4), which represent two different algorithms used to implement optimization) are listed in tables specifically. In addition, to better validate the effect of the decontamination step, the proposed method is also compared with the method without optimization step (Ours(baseline)) in the same tracking framework. As higher AOR value and lower ACLE value represent better performance, conclude from data in tables we can see that the method optimized with $J_{1}$ objective function can achieve a higher AOR value, and the method optimized with $J_{2}$ objective function can achieve a lower ACLE value. However compared to the tracking method without optimization step, the performance of which with both these two objective functions have been enhanced a lot.

Overall, the proposed adaptive model realizes better or at least comparative performance in comparison with the other methods. Generally speaking, for many challenging videos, our method 


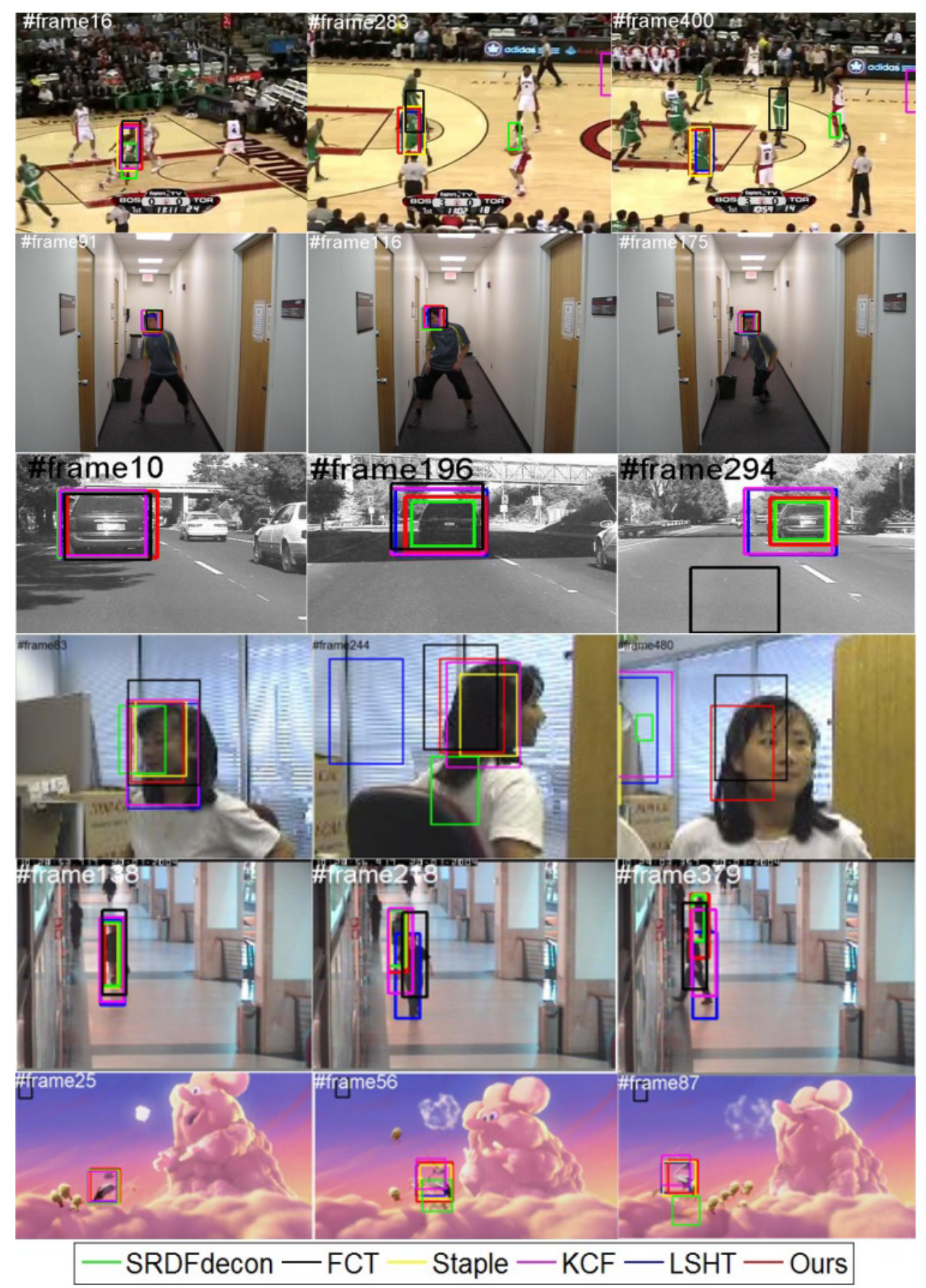

Fig 8 Tracking results comparison of the tested videos on some key frames, where the tracking results are represented as a bounding box. Different color represents different tracker.

is qualified to provide the accurate locations of the target.

For more informative comparison, the qualitative tracking results obtained by multiple algorithms on the selected sequences are illustrated in Fig.8 and Fig.9. We can conclude that our method outperforms most of the other trackers against tracking challenges including complex 


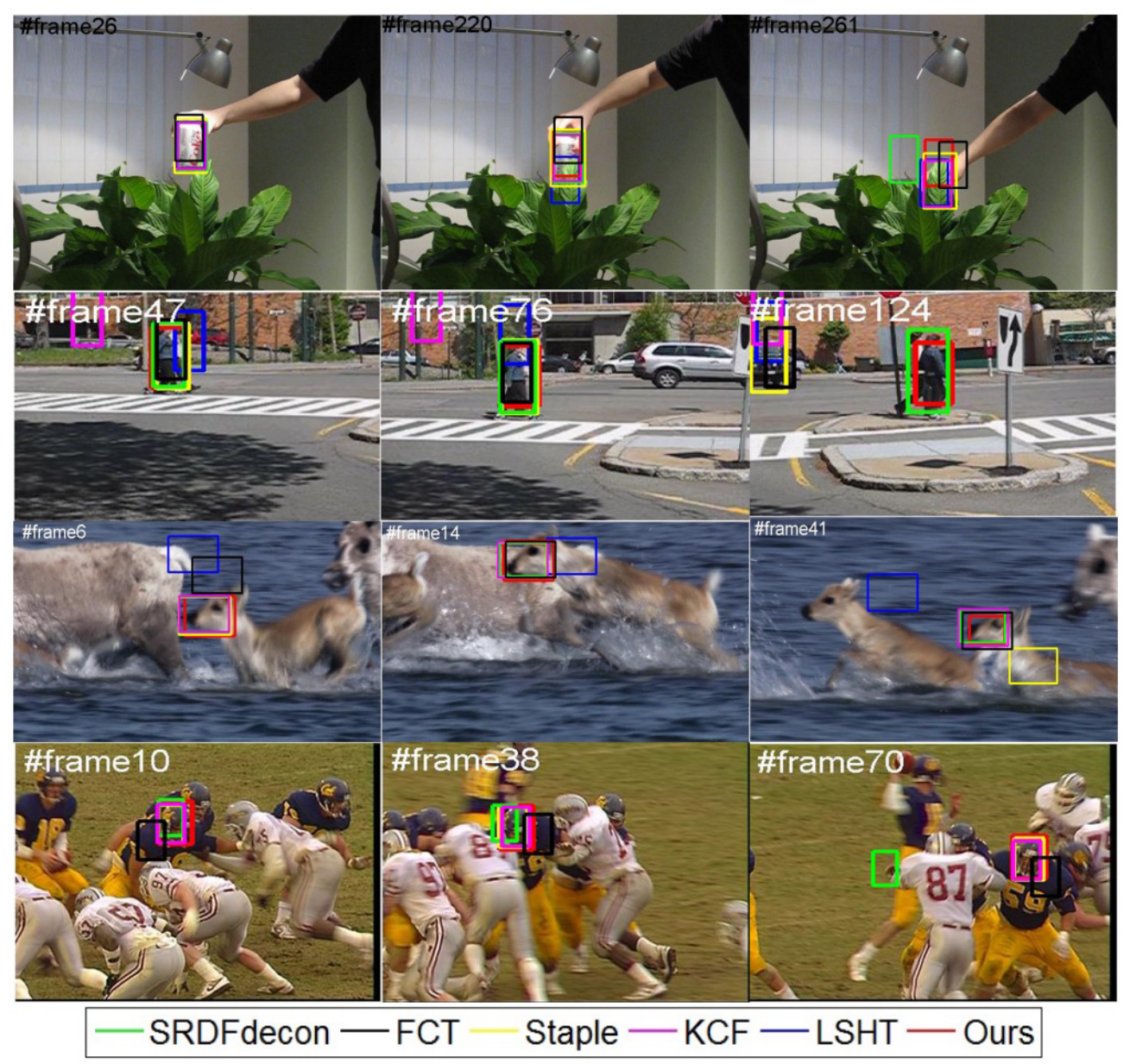

Fig 9 Tracking results comparison of the tested videos on some key frames, where the tracking results are represented as a bounding box. Different color represents different tracker.

Table 2 Tracking results on AOR, where $\operatorname{DFT}\left(J_{1}\right)$ and $\operatorname{DFT}\left(J_{2}\right)$ are the data for the proposed tracking employ different object functions shown in formula (2) and (4)

\begin{tabular}{lllllllll}
\hline AOR & LSHT & FCT & KCF & Staple & SRDCFdecon & $\begin{array}{l}\text { Ours } \\
\text { (baseline) }\end{array}$ & $\begin{array}{l}\text { Ours } \\
\left(J_{1}\right)\end{array}$ & $\begin{array}{l}\text { Ours } \\
\left(J_{2}\right)\end{array}$ \\
\hline BOY & 0.3393 & 0.63 & 0.65 & 0.74 & 0.83 & 0.74 & 0.73 & 0.73 \\
Basketball & 0.4363 & 0.23 & 0.68 & 0.44 & 0.42 & 0.58 & 0.61 & 0.62 \\
Car4 & 0.2097 & 0.24 & 0.59 & 0.79 & 0.87 & 0.45 & 0.71 & 0.70 \\
Coke & 0.1589 & 0.36 & 0.39 & 0.58 & 0.56 & 0.62 & 0.63 & 0.69 \\
Couple & 0.1778 & 0.48 & 0.22 & 0.5 & 0.73 & 0.6 & 0.64 & 0.64 \\
Deer & 0.1081 & 0.67 & 0.42 & 0.68 & 0.8 & 0.76 & 0.68 & 0.78 \\
Football1 & 0.5834 & 0.17 & 0.48 & 0.76 & 0.48 & 0.74 & 0.76 & 0.76 \\
Girl & 0.2499 & 0.36 & 0.51 & 0.55 & 0.63 & 0.42 & 0.45 & 0.52 \\
Walking2 & 0.3788 & 0.28 & 0.38 & 0.76 & 0.808 & 0.46 & 0.67 & 0.65 \\
Bird2 & 0.5421 & 0.1 & 0.58 & - & - & 0.66 & 0.66 & 0.71 \\
\hline Average & 0.32 & 0.35 & 0.49 & 0.64 & 0.67 & 0.60 & 0.65 & 0.68 \\
\hline
\end{tabular}


Table 3 Tracking results on ACLE, where $\operatorname{DFT}\left(J_{1}\right)$ and $\operatorname{DFT}\left(J_{2}\right)$ are the data for the proposed tracking employ different object functions shown in formula (2) and (4)

\begin{tabular}{lllllllll}
\hline ACLE & LSHT & FCT & KCF & Staple & SRDCFdecon & $\begin{array}{l}\text { Ours } \\
\text { (baseline) }\end{array}$ & $\begin{array}{l}\text { Ours } \\
\left(J_{1}\right)\end{array}$ & $\begin{array}{l}\text { Ours } \\
\left(J_{2}\right)\end{array}$ \\
\hline BOY & 15.67 & 6.07 & 1.97 & 2.54 & 1.5 & 1.16 & 1.06 & 1.03 \\
Basketball & 70.64 & 77.4 & 5.02 & 11.52 & 18.35 & 16.51 & 7.66 & 12.55 \\
Car4 & 87.71 & 37.7 & 9.58 & 12.1 & 1.56 & 17.07 & 3.17 & 3.23 \\
Coke & 35.59 & 12 & 12.67 & 9.36 & 13.35 & 7.6778 & 5.93 & 5.05 \\
Couple & 64.33 & 31.9 & 45 & 4.3 & 3.3 & 3.077 & 2.58 & 2.49 \\
Deer & 41.33 & 4.4 & 11.6 & 10.5 & 2.7 & 3.86 & 2.49 & 3.66 \\
Football1 & 5.92 & 16.4 & 3.25 & 2.3 & 6.04 & 2.363 & 1.69 & 1.55 \\
Girl & 24.45 & 13 & 8.39 & 7.9 & 2.3 & 11.77 & 9.85 & 8.44 \\
Walking2 & 2.79 & 23.5 & 6.66 & 1.8 & 0.88 & 3.46 & 1.09 & 1.09 \\
Bird2 & 17.15 & 45.82 & 7.67 & - & 8.32 & 9.69 & 7.88 & 6.16 \\
\hline Average & 36.56 & 26.8 & 11.18 & 6.9 & 5.83 & 7.66 & 4.34 & 4.52 \\
\hline
\end{tabular}

background, fast motion, occlusion, deformation and so on. The success of our method mainly due to two factors: the first one is that the features used in the tracking process are different between video sequences, and every feature subset is corresponding to the character of current video sequences. With high discriminative visual model, the accuracy of tracker is sure to be enhanced. The second factor is: SMC based framework is employed to update tracking parameters and feature pool, target deformation and abrupt changes are able to be handled in the tracking process. As a result, the stability and adaptability of the tracking are well guaranteed.

\section{Conclusion}

In this paper, a novel scheme is proposed to improve the adaptability and robustness of visual models. Specifically, the visual modeling is converted into an optimization problem, where the optimal solution is determined by applying the classical evolutionary algorithm. By applying the determined adaptive visual model for specific video sequence, it helps to provide a new view for the visual modeling problem. The Genetic Algorithm is employed as an example due to its strong generalization capability, other evolutionary algorithms such as Artificial immune algorithm, Simulated annealing algorithm can also be employed. Although in our experiments only color, edge and texture features are employed, more visual cues such as motion and Haar-like features can also be added. For future work, we will integrate more features and other existing evolutionary algorithms to further improve the performance of the proposed trackers.

\section{Acknowledgement}

This work is supported by the National Natural Science Foundation of China under Grant No. 61300099, the China Postdoctoral Science Foundation funded project under Grant No. 2015M570261, and the Science and Technology Development Plan of Jilin Province under grant No. 20170101144JC, the open fund of China key Laboratory of Symbolic Computation and Knowledge Engineering of Ministry of Education under Grand No. 93K172016K14. 


\section{References}

1 S. Ali and M. Shah, "Floor fields for tracking in high density crowd scenes," in European Conference on Computer Vision, 1-14 (2008).

2 D. A. Forsyth and J. Ponce, "Computer vision: A modern approach," 14(1), 133149 (2002).

3 F. S. F. M. W. J. V. D. Danelljan, Martin Khan, "Adaptive color attributes for real-time visual tracking," in IEEE Conference on Computer Vision and Pattern Recognition, 1090-1097 (2014).

4 P. Zhao, H. Zhu, H. Li, et al., "A directional-edge-based real-time object tracking system employing multiple candidate-location generation," IEEE Transactions on Circuits and Systems for Video Technology 23(3), 503-517 (2013).

5 A. R. Zamir, A. Dehghan, and M. Shah, "Gmcp-tracker: Global multi-object tracking using generalized minimum clique graphs," (2012).

6 S. Zhang, H. Yao, X. Sun, et al., "Sparse coding based visual tracking: Review and experimental comparison," Pattern Recognition 46(7), 1772-1788 (2013).

7 G. Yuan, M. Xue, P. Zhou, et al., PCA-based adaptive particle filter for tracking (2010).

8 R. Ramezani, P. Angelov, and X. Zhou, "A fast approach to novelty detection in video streams using recursive density estimation," in Intelligent Systems, 2008. International IEEE Conference, 14-2 - 14-7 (2008).

9 J. F. Henriques, C. Rui, P. Martins, et al., "High-speed tracking with kernelized correlation filters," IEEE Transactions on Pattern Analysis and Machine Intelligence 37(3), 583-596 (2015).

10 N. Dalal and B. Triggs, "Histograms of oriented gradients for human detection," IEEE Computer Society Conference on Computer Vision and Pattern Recognition. IEEE Computer Society Conference on Computer Vision and Pattern Recognition 1(12), 886-893 (2005).

11 K. Zhang, L. Zhang, and M. H. Yang, "Real-time compressive tracking," in European Conference on Computer Vision, 864-877 (2012).

12 R. Lienhart and J. Maydt, "An extended set of haar-like features for rapid object detection," 1, I-900-I-903 vol.1 (2002).

13 M. Spengler and B. Schiele, "Towards robust multi-cue integration for visual tracking," $M a-$ chine Vision and Applications 14(1), 50-58 (2003).

14 P. Perez, J. Vermaak, and A. Blake, "Data fusion for visual tracking with particles," Proceedings of the IEEE 92(3), 495-513 (2004).

15 H. Wang, D. Suter, K. Schindler, et al., "Adaptive object tracking based on an effective appearance filter," IEEE Transactions on Pattern Analysis and Machine Intelligence 29(9), 1661-1667 (2007).

$16 \mathrm{Y}$. Wu and T. S. Huang, "Robust visual tracking by integrating multiple cues based on coinference learning," International Journal of Computer Vision 58(1), 55-71 (2004).

17 Q. Bai, Z. Wu, S. Sclaroff, et al., "Randomized ensemble tracking," Conference on Computer Vision and Pattern Recognition. , 2040-2047 (2013).

18 X. W. Lijun Wang, Wanli Ouyang and H. Lu., "Stct: Sequentially training convolutional networks for visual tracking," Conference on Computer Vision and Pattern Recognition. , 1063-6919 (2016). 
19 H. Grabner, M. Grabner, and H. Bischof, "Real-time tracking via on-line boosting," in British Machine Vision Conference 2006, Edinburgh, Uk, September, 47-56 (2006).

20 D. E. Goldberg, Genetic algorithms in search, optimization, and machine learning, AddisonWesley Pub. Co., (1989).

21 Y. Wu, J. Lim, and M. H. Yang, "Object tracking benchmark," IEEE Transactions on Pattern Analysis and Machine Intelligence 37(9), 1834-48 (2015).

22 M. Kristan, A. Leonardis, J. Matas, et al., The Visual Object Tracking VOT2016 Challenge Results, Springer International Publishing (2016).

23 T. Darrell and K. L. Grauman, "Matching sets of features for efficient retrieval and recognition," Massachusetts Institute of Technology (2006).

24 D. B. Fogel, Evolutionary algorithms in theory and practice, Oxford Univ. Pr (1998).

25 S. Das and P. N. Suganthan, "Differential evolution: A survey of the state-of-the-art," IEEE Transactions on Evolutionary Computation 15(1), 4-31 (2011).

26 H. Tang, S. Xue, and C. Fan, "Differential evolution strategy for structural system identification," Computers and Structures 86(2122), 2004-2012 (2008).

27 R. A. F. S. F.R.S., "The use of multiple measurements in taxonomic problems," Annals of Eugenics 7(2), 179188 (1936).

28 M. Y. S. W. Yuru Wang, Qiaoyuan Liu, "Large margin classifier-based ensemble tracking," Journal of Electronic Imaging 25(4), 043006 (2016).

29 A. Doucet, S. Godsill, and C. Andrieu, "On sequential monte carlo sampling methods for bayesian filtering," Statistics and Computing 10(3), 197-208 (2003).

30 A. W. Smeulders, D. M. Chu, R. Cucchiara, et al., "Visual tracking: An experimental survey.," IEEE Transactions on Pattern Analysis and Machine Intelligence 36(7), 1442-68 (2014).

31 K. Z., "Tracking-learning-detection," IEEE Transactions on Pattern Analysis and Machine Intelligence 34(7), 1409-1422 (2012).

32 E. M., “The pascal visual object classes (voc) challenge," International Journal of Computer Vision 88(2), 303-338 (2010).

33 Y. M. H. Wu Y, Lim J, “Online object tracking: A benchmark," IEEE Conference on Computer Vision and Pattern Recognition , 2411-2418 (2013).

34 B. S. Babenko B, Yang M H, "Visual tracking with online multiple instance learning," Computer Vision and Pattern Recognition, 983-990 (2009).

35 J. Kwon and K. M. Lee, "Visual tracking decomposition," in IEEE Conference on Computer Vision and Pattern Recognition, 1269-1276 (2010).

36 T. P. H. S. Hare S, Saffari A, "Struck: Structured output tracking with kernels," International Conference on Computer Vision. , 263-270 (2011).

37 Z. W, "Robust object tracking via sparsity-based collaborative model," IEEE Conference on Computer Vision and Pattern Recognition , 1838-1845 (2012).

38 S. He, Q. Yang, R. W. H. Lau, et al., "Visual tracking via locality sensitive histograms," in Cvpr, IEEE Computer Society Conference on Computer Vision and Pattern Recognition. IEEE Computer Society Conference on Computer Vision and Pattern Recognition, 24272434 (2013). 
39 K. Zhang, L. Zhang, and M. H. Yang, "Fast compressive tracking.," IEEE Transactions on Pattern Analysis and Machine Intelligence 36(10), 2002-15 (2014).

40 L. Bertinetto, J. Valmadre, and Golodetz, "Staple:complementary learners for real-time tracking," The IEEE Conference on Computer Vision and Pattern Recognition (2016).

41 K. F. S. e. a. Danelljan M, Hager G, "Adaptive decontamination of the training set: A unified formulation for discriminative visual tracking," IEEE Conference on Computer Vision and Pattern Recognition (2016).

42 M. Danelljan, G. Hager, F. S. Khan, et al., "Learning spatially regularized correlation filters for visual tracking," in IEEE International Conference on Computer Vision, 4310-4318 (2016).

Yuru Wang received $\mathrm{PhD}$ degree in Department of Computer Science and Technology from Harbin Institute of Technology, China in 2010. Her current research interests include computer visions and pattern recognition

Qiaoyuan Liu received the bachelors degree in Department of Computer Science and Technology from the Northeast University, Shenyang, China, in 2014. Currently, she is pursing the M.S. degree in Department of Computer Science and Technology from Northeast Normal University, Changchun, China. Her current research interests is visual tracking.

Minghao Yin received $\mathrm{PhD}$ degree in College of Computer Science and Technology, Jilin University, China in 2008. His current research interests mainly include automated planning, automated reasoning, semantic web and approximate reasoning.

Biographies and photographs of the other authors are not available.

\section{List of Figures}

1 The salient features in different video examples.

2 Overview of the proposed decontaminating feature based tracker.

3 Approximate the optional solution by the binary evolutionary algorithm.

4 Schematic diagram of objective function optimization, in which the green bounding boxes in the first pi

5 The curves of the parameter settings on tracking performance.

$6 \quad$ Average success plot and precision plot on OTB2015.

$7 \quad$ Average expected overlap plot on VOT2016(a). Performance rank between our method and other state-c

8 Tracking results comparison of the tested videos on some key frames, where the tracking results are rep

9 Tracking results comparison of the tested videos on some key frames, where the tracking results are rep

\section{List of Tables}

1 Tracking sequences used in our experiments

2 Tracking results on $\operatorname{AOR}$, where $\operatorname{DFT}\left(J_{1}\right)$ and $\operatorname{DFT}\left(J_{2}\right)$ are the data for the proposed tracking employ di

3 Tracking results on $\operatorname{ACLE}$, where $\operatorname{DFT}\left(J_{1}\right)$ and $\operatorname{DFT}\left(J_{2}\right)$ are the data for the proposed tracking employ 